\title{
A novel readiness assessment framework for the application of artificial intelligence in the healthcare sector
}

\author{
L.R.A.B. Jayaratne ${ }^{1 *}$, V.H.W. Dissanayake ${ }^{2}$ \\ 1 National Hospital of Sri Lanka, Colombo, Sri Lanka \\ 2 Postgraduate Institute of Medicine, University of Colombo, Sri Lanka \\ *jayaratne.Irab2017@pgim.cmb.ac.lk \\ iD https://orcid.org/0000-0002-0791-3334 \\ ABSTRACT
}

\begin{abstract}
Introduction: When introducing new technology, pre-implementation readiness assessment is not a new concept. Though published data on readiness assessment on electronic health records, health information technology, and eHealth are abundant, no studies have been conducted on readiness assessment on the application of artificial intelligence (AI) in healthcare institutions. Moreover, the structure and composition of the assessment tools vary with adopting technology and perspectives of the institution considered.

Methods: In this study, a novel framework was developed to assess the readiness for artificial intelligence in healthcare institutions. By extending this framework, a novel employee readiness assessment tool and structural readiness assessment tool were developed to assess the institutional readiness for artificial intelligence-based ophthalmological diagnosis support systems. Both tools were tested and validated. Using these tools, institutional readiness was measured at National Eye Hospital of Sri Lanka (NEH).

Results: $27 \%$ of employees of NEH were ready to accept Al-based Ophthalmological diagnosis support systems at the hospital Out-Patient Department (OPD), while there was only $8 \%$ structural readiness at the hospital.

Conclusions: This study presents the first readiness assessment framework for the healthcare sector with validated tools to assess the institutional readiness for artificial intelligence-based ophthalmological diagnosis support systems. The developed framework and tools can be adapted to assess the readiness for artificial intelligence in any healthcare institution.
\end{abstract}

Keywords: Artificial Intelligence, Pre-implementation, Readiness assessment, Healthcare, Ophthalmology 